\title{
SPLUNC1 Promotes Lung Innate Defense Against Mycoplasma pneumoniae Infection in Mice
}

\author{
Fabienne Gally, ${ }^{*}$ Y. Peter Di, ${ }^{\dagger}$ Sean K. Smith, ${ }^{*}$ \\ Maisha N. Minor, ${ }^{*}$ Yang Liu, ${ }^{\dagger}$ Donna L. Bratton, ${ }^{\ddagger}$ \\ S. Courtney Frasch, ${ }^{\ddagger}$ Nicole M. Michels, ${ }^{*}$ \\ Stephanie R. Case, ${ }^{*}$ and Hong Wei Chu* \\ From the Departments of Medicine, ${ }^{*}$ and Pediatrics, ${ }^{\ddagger}$ National \\ Jewish Health, Denver, Colorado; and the Department of \\ Environmental and Occupational Health, ${ }^{\dagger}$ University of \\ Pittsburgh, Pittsburgh, Pennsylvania
}

Short palate, lung, and nasal epithelium clone 1 (SPLUNC1) protein is highly expressed in normal airways, but is dramatically decreased in allergic and cigarette smoke exposure settings. We have previously demonstrated SPLUNC1 in vitro antibacterial property against Mycoplasma pneumoniae (Mp). However, its in vivo biological functions remain unclear. The objectives of this study were to determine the in vivo functions of SPLUNC1 following bacterial (eg, Mp) infection, and to examine the underlying mechanisms. We generated SPLUNC1deficient mice and utilized transgenic mice overexpressing human SPLUNC1 exclusively within the airway epithelium. These mice were infected with Mp and, twenty-four hours post infection, their host defense responses were compared to littermate controls. Mp levels and inflammatory cells increased in the lungs of SPLUNC1 ${ }^{-/-}$mice as compared to wild type controls. SPLUNC1 deficiency was shown to contribute to impaired neutrophil activation. In contrast, mice overexpressing hSPLUNC1 exclusively in airway epithelial cells demonstrated lower $\mathrm{Mp}$ levels. Furthermore, neutrophil elastase activity was significantly increased in mice overexpressing hSPLUNC1. Lastly, we demonstrated that SPLUNC1 enhanced Mp-induced human neutrophil elastase (HNE) activity, and HNE directly inhibited the growth of $\mathrm{Mp}$. Our findings demonstrate a critical $i n$ vivo role of SPLUNC1 in host defense against bacterial infection, and likely provide a novel therapeutic approach to restore impaired lung innate immune responses to bacteria in patients with chronic lung diseases. (Am J Pathol 2011, 178:2159-2167; DOI: 10.1016/j.ajpath.2011.01.026)
Located at the interface between the host and the environment, the airway epithelium is the first line of host defense against environmental hazards such as pathogens. Because airway mucosal innate immunity acts quickly to prevent the host from the damaging effects of pathogens, it is essential that the normal functions of the airway epithelium be maintained. ${ }^{1}$ Airway epithelial cells exert their host defense activity by recognizing the invading microbes and subsequently producing various mediators such as cytokines (eg, IL-8 and granulocyte macrophage colony-stimulating factor), mucins (eg, MUC5AC), and antimicrobial substances including peptides ( $\beta$-defensins and LL-37) and proteins (lysozyme and lactoferrin). ${ }^{2}$

Despite previous efforts in investigating airway mucosal immunity, respiratory bacterial and viral infections continue to pose a substantial challenge to health care in patients with various lung diseases. Therefore, more research work is imperative to define the in vivo functions of key molecules in the airway epithelial cell defense against pathogens. It has recently been demonstrated that airway epithelial cells in mice and human beings express high levels of short palate, lung, and nasal epithelium clone 1 (SPLUNC1). $^{3,4}$ SPLUNC1 is a member of the PLUNC family, which encompasses 10 genes located on human chromosome 20q11. ${ }^{5}$ Because SPLUNC1 has been predicted to share structural similarities with bactericidal permeability-increasing protein, it was hypothesized that it may be involved in host defense against bacterial infection. However, this hypothesis has not been rigorously tested, in part owing to lack of a SPLUNC1-deficient animal model. Nonetheless, in a previous study, ${ }^{4}$ it was demonstrated that recombinant mouse SPLUNC1 protein exerts in vitro antimicrobial properties. Moreover, Zhou et $\mathrm{al}^{6}$ demonstrated that SPLUNC1 inhibited Pseudomonas aeruginosa growth and the overall process of EpsteinBarr virus replication. In addition to its potential antimi-

Supported by grants RO1HL088264 (H.W.C.) and R01HL091938 (Y.P.D.) from the National Heart, Lung, and Blood Institute, National Institutes of Health.

Accepted for publication January 13, 2011.

Address reprint requests to Hong Wei Chu, M.D., National Jewish Health, 1400 Jackson St, Rm A639, Denver, CO 80206. E-mail: chuhw@ njhealth.org. 
crobial function, SPLUNC1 regulates airway surface liquid volume in vitro. ${ }^{7}$

Each year, approximately 2 million pneumonia cases due to infection with Mycoplasma pneumoniae (Mp), including 100,000 hospitalizations, are reported in the United States. ${ }^{8} \mathrm{Mp}$ is an atypical bacterium that lacks a cell wall and relies on adhesion to host cells for colonization and infection, which is generally restricted to airway epithelium. Mp can be observed in the airways of $45 \%$ of adults with chronic asthma. ${ }^{9}$ Eradication of $\mathrm{Mp}$ using antibiotic therapy is difficult. Previous studies ${ }^{10}$ have demonstrated that clarithromycin therapy in patients with asthma with $\mathrm{Mp}$ improved lung function; however, Mp persisted in the airways. Thus, it is important to find alternative therapies such as endogenous antimicrobial substances to effectively attenuate or eliminate $\mathrm{Mp}$ infection without causing antibiotic resistance.

The objective of the present study was to determine the in vivo function of SPLUNC1 in lung bacterial infection. SPLUNC $1^{-1-}$ mice were generated, and their response to $\mathrm{Mp}$ infection was analyzed in comparison with that of littermate control mice. To examine the role of human SPLUNC1 (hSPLUNC1), transgenic mice overexpressing hSPLUNC1 exclusively within the airway epithelium were also generated and infected with $\mathrm{Mp}$. To further understand the mechanisms of SPLUNC1 in host defense against $\mathrm{Mp}$ infection, human peripheral blood neutrophil culture was used. It was observed that SPLUNC1 is necessary and sufficient to confer host defense against $\mathrm{Mp}$ infection in vivo. Moreover, in the presence of $\mathrm{Mp}$ infection, SPLUNC1 seems to enhance neutrophil innate immune functions.

\section{Materials and Methods}

\section{Generation of SPLUNC1 Gene-Deficient Mice}

The SPLUNC1 targeting vector was generated from the substitution of the protein coding sequence (CCDS16925.1) by the neomycin resistance gene and the Escherichia coli $\beta$-galactosidase gene (Velocigene, Regeron Pharmaceuticals, Inc., Tarrytown, NY) (Figure 1, $A$ and $B)$. The selection of embryonic stem cell clones containing the targeting vector was performed using quantitative PCR for loss of allele as described previously. ${ }^{11}$ Embryonic stem cells were electroporated with the targeting vector, selected, and expanded (Knockout Mouse Project, University of California Davis, Davis, CA). Embryonic stem cell clones were injected into $\mathrm{B} 6(\mathrm{Cg})-\mathrm{Tyr}^{\mathrm{C}-2 \mathrm{~J}} / \mathrm{J}$ (B6 albino) blastocysts, which were subsequently implanted in pseudopregnant ICR females in our Mouse Genetics Core facility. Resulting chimeras were crossed to B6(Cg)-Tyr ${ }^{\mathrm{C}-2 \mathrm{~J} / \mathrm{J}}$ (B6 albino) mice to establish germline transmission. SPLUNC1 $1^{+/}$F1 mice were intercrossed, and F2 breeding pairs were established from SPLUNC $1^{-1-}$ and SPLUNC $1^{+/+}$mice. To determine the mouse genotype, dual PCR with the primer sets for mouse SPLUNC1 (forward, 5'-CCACACAGAGGGACTTGGTT-3', and reverse, 5'-TCAGGCTTGGGTATGTGGTC-3') or neomycin (forward, 5'-TCATTCTCAGTATT-
GTTTTGCC-3', and reverse, 5'-TACTTTCTCGGCAGGAGCAAGGTG-3') was performed using genomic DNA from mouse tail clips. Also confirmed was the "loss of native allele" using PCR. Two target-specific primer sets were designed that corresponded to the upstream and downstream end of each targeted deletion (Figure 1B).

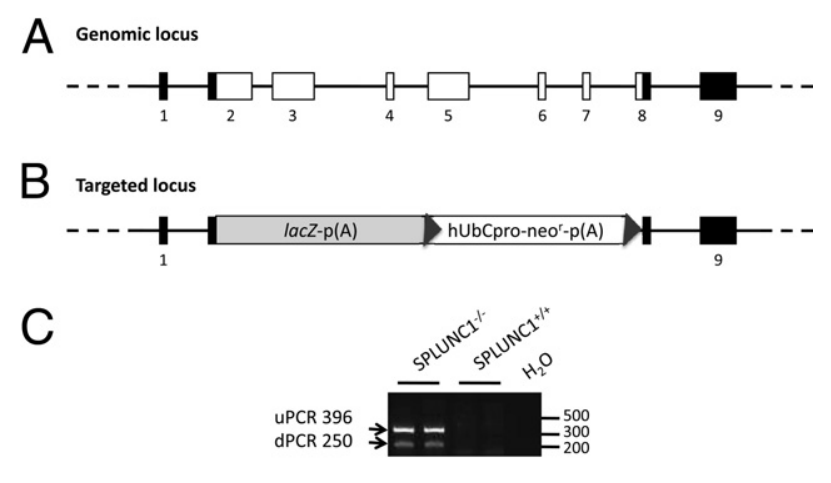

D

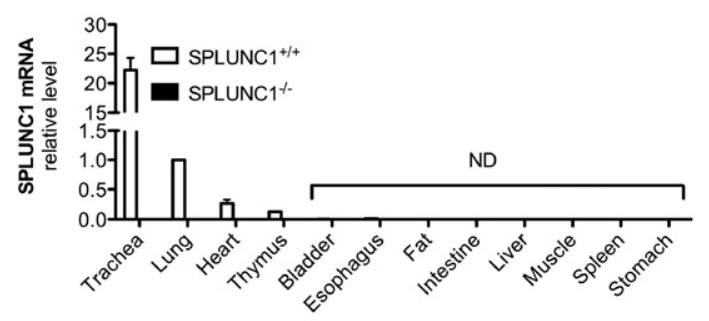

E

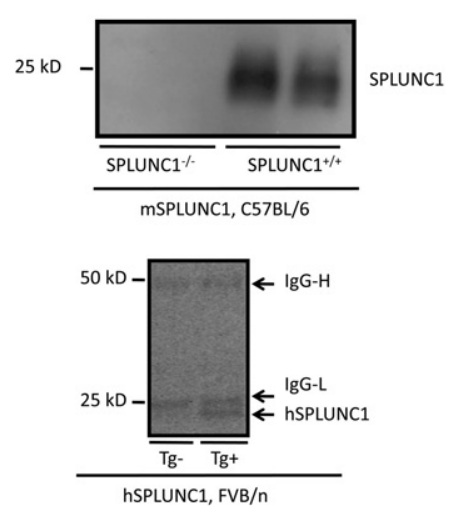

Figure 1. Generation of SPLUNC1 ${ }^{-1-}$ mice and detection of SPLUNC1 in SPLUNC1 $1^{-/-}$and hSPLUNC1 transgenic mice. A: Schema of the SPLUNC1 genomic locus. Black squares, noncoding exons; white squares, coding exons. B: Schema of the targeted locus. Homologous recombination resulted in replacement of SPLUNC1 genomic region (chromosome 2 deletion from $153,969,486$ to $153,973,826$ ) via expression-selection cassette containing lacZneomycin resistance genes. lac $Z$, coding sequence for $\beta$-galactosidase from the E. coli lac $Z$ gene. $\mathrm{p}(\mathrm{A})$, polyadenylation signal; neo', coding sequence for neomycin phosphotransferase; hUbCpro, promoter from human ubiquitin C gene; uPCR, upstream PCR; dPCR, downstream PCR. C: PCR confirmation of targeting construct orientation in chromosome 2 of SPLUNC1 $1^{-1-}$ mice. UPCR, detection of $\beta$-galactosidase (upstream) in mouse genomic DNA; dPCR, detection of neomycin (downstream) in mouse genomic DNA. No amplification was detected in SPLUNC1 $1^{+/+}$mice. D: Tissue SPLUNC1 mRNA levels. Various tissues were obtained from SPLUNC1 ${ }^{+/+}$or SPLUNC1 $1^{-/-}$mice $(n=$ 3 per group). Data are given as mean (SEM) and normalized to wild-type lung tissue expression. ND, not detectable. E: mSPLUNC1 protein was detected using Western blot analysis using sheep anti-mouse SPLUNC1 antibody in BAL fluid (30 $\mu \mathrm{L}$ per lane) from SPLUNC1 ${ }^{+/+}$but not SPLUNC1 $^{-/-}$mice F: hSPLUNC1 protein was detected using Western blot analysis using mouse anti-human SPLUNC1 antibody in BAL fluid (30 $\mu$ Ler lane) from hSPLUNC1 transgene-positive $(\mathrm{Tg}+)$ but not transgene-negative $(\mathrm{Tg}-)$ mice. The 50 and $25 \mathrm{kDa}$ bands represent mouse IgG heavy (IgG-H) and IgG light (IgG-L) chains, respectively. 
PCR amplicons were detected only in SPLUNC1-deficient animals, confirming the gene substitution by the targeted vector and its correct insertion (Figure 1C).

\section{Generation of Scgb1a1-hSPLUNC1 Transgenic Mice}

Constitutive expression of hSPLUNC1 protein in the mouse respiratory epithelium was achieved through generation of mice that harbored a transgene composed of hSPLUNC1 cDNA under the transcriptional control of the mouse Scgb1a1 (also known as Clara cell secretory protein) promoter. Total human airway epithelial cell RNA was used as a template for RT-PCR amplification of SPLUNC1 cDNA, $0.78 \mathrm{~kb}$. Human SPLUNC1 cDNA product was amplified, gel purified, cloned using the pCR2.1TOPO cloning system (Invitrogen Corp., Carlsbad, CA), and sequenced. A 9.6-kb Scgb1a1 genomic fragment in pUC19 was modified using site-directed mutagenesis to include a Notl site upstream of the Scgb1a1 translational start site, and resulted in generation of pNotl-9.Scgb1a1. The hSPLUNC1 cDNA was cloned into the Notl site of pNotl-9.6ScgbB1a1, and the construct was verified at restriction enzyme digestion and sequencing. The 10.38-kb Scgb1a1-hSPLUNC1 cDNA fragment was isolated via digestion with Sphl and transgenic mice generated via microinjection into $\mathrm{FVB} / \mathrm{n}$ mouse oocytes. One of 19 offspring was positive for the transgene, as assessed at PCR genotyping and confirmed at Southern blot analyses of tail DNA. Subsequently, the genotype was determined using PCR. The endogenous Scgb1a1 gene and the Scgb1a1-hSPLUNC1 transgene were distinguished as 450- and 545-bp amplicons using a mouse Scgb1a1 promoter-specific forward primer (5'-GTTGGCAAGTCTACAGTTGC-3') and a SPLUNC1 coding region forward primer (5'-GACGTCAGTGATTCCTGGCC-3'), in combination with a Scgb1a1 intron 1-specific reverse primer (5'-GAAAGAGACCCTGGGCACTCA-3').

\section{Mp Infection in Mice}

SPLUNC $1^{-1-}$ mice and littermate control mice on a C57BL/6 background (8 to 12 weeks) were bred in our Biological Resources Center. Scgb1a1 hSPLUNC1 transgenic mice and littermate control mice on an FVB/n background were bred at the University of Pittsburgh mouse facility. All experimental animals were performed in accordance with protocols approved by the Institutional Animal Care and Use Committees of both National Jewish Health and the University of Pittsburgh.

Mice were anesthetized via intraperitoneal injection of tribromoethanol (Avertin; Sigma-Aldrich, St. Louis, MO), $0.25 \mathrm{~g} / \mathrm{kg}$ body weight, and inoculated intranasally using $50 \mu \mathrm{L} \mathrm{Mp}$ at $1 \times 10^{8}$ colony-forming units (CFUs) or 50 $\mu \mathrm{L}$ saline solution. After 24 hours, mice were sacrificed to examine bronchoalveolar lavage (BAL) cell profiles, lung Mp load, SPLUNC1 expression, lung tissue histopathologic features, and neutrophil elastase activity.

\section{BAL and Lung Tissue Processing}

Mice were euthanized via intraperitoneal injection of pentobarbital sodium, $200 \mathrm{mg} / \mathrm{kg}$, and tracheotomized. The lungs were lavaged once with $1 \mathrm{~mL}$ PBS. Cell-free BAL fluid was stored at $-80^{\circ} \mathrm{C}$ for SPLUNC1 Western blot analysis and neutrophil elastase activity. BAL cell cytospins were stained with the Diff-Quick Stain Kit (IMEB, Inc., San Marcos, CA) for cell differential counts. The left lung lobe was homogenized in SP-4 broth to perform Mp culture as previously described. ${ }^{12}$ Right lung lobes were used for histopathology and RNA extraction.

\section{Real-Time RT-PCR}

Real-time RT-PCR was performed to determine mouse and human SPLUNC1 mRNA levels as described previously. ${ }^{12}$ Unless otherwise noted, $18 \mathrm{~S}$ rRNA was used as the housekeeping gene to normalize the expression of the gene of interest. The threshold cycle was recorded for each sample to reflect mRNA expression levels. The comparative threshold cycle method was used to demonstrate the relative expression level of the gene of interest.

\section{Western Blot Analysis of Mouse and Human SPLUNC1 Protein}

Western blot analysis was used to quantify SPLUNC1 protein. In brief, $30 \mu \mathrm{L}$ BAL fluid was electrophoresed on $10 \%$ SDS-polyacrylamide gel electrophoresis, transferred onto nitrocellulose membrane, blocked with the Western blocking buffer, and incubated with a sheep anti-mouse SPLUNC1 or a mouse anti-human SPLUNC1 antibody (R\&D Systems, Inc., Minneapolis, MN) overnight at $4^{\circ} \mathrm{C}$. After washes in PBS with $0.1 \%$ Tween 20 , the membranes were incubated with an anti-sheep IgG or anti-mouse IgG conjugated to horseradish peroxidase.

\section{SPLUNC1 Antimicrobial Activity Assay}

The antimicrobial effect of SPLUNC1 was tested by incubating $\mathrm{Mp}, 10^{5} \mathrm{CFU} / \mathrm{mL}$, with recombinant hSPLUNC1, $10 \mu \mathrm{g} / \mathrm{mL}$, or bovine serum albumin (BSA), $10 \mu \mathrm{g} / \mathrm{mL}$, in a 96-well plate for 24 hours at $35^{\circ} \mathrm{C}$. Selection of this SPLUNC1 protein dosage was based on SPLUNC1 protein measurements in airway epithelial lining fluid in healthy individuals $(n=12)$ with no history of respiratory disease or cigarette smoking and who had normal pulmonary function (forced expiratory volume in 1 minute $>80 \%$ ). Mean (SD) age of the seven men and five women was 33.1 (3.0) years. SPLUNC1 protein in BAL fluid in healthy human subjects was measured using a direct SPLUNC1 enzyme-linked immunosorbent assay. After normalization of dilution factor using the serum urea-BAL urea ratio, SPLUNC1 protein concentration in airway epithelial lining fluid was calculated at $5.3(2.1) \mu \mathrm{g} / \mathrm{mL}$.

To determine whether SPLUNC1 exerts antimicrobial effects through interfering bacterial adherence, the $\mathrm{Mp}$ specific P1 adhesin mRNA level was measured. In brief, $\mathrm{Mp} 1 \times 10^{6} \mathrm{CFU} / \mathrm{mL}$ was incubated with recombinant hSPLUNC1 protein, $10 \mu \mathrm{g} / \mathrm{mL}$, or BSA, $10 \mu \mathrm{g} / \mathrm{mL}$, in 
six-well plates for 24 hours. Bacterial RNA was extracted, and real-time PCR was performed to quantify P1 adhesin mRNA by normalizing the data to $16 \mathrm{~S}$ rRNA levels.

\section{Lung Histopathology}

Lungs were harvested and fixed in 10\% phosphate-buffered formalin overnight, then dehydrated in $70 \%$ ethanol as described previously. ${ }^{13}$ Lung tissues were embedded in paraffin, cut in $4-\mu \mathrm{m}$ thick sections, and stained with $\mathrm{H} \& \mathrm{E}$. The stained lung sections were evaluated in a double-blind fashion under a light microscope using a histopathologic inflammatory scoring system as described previously in a hamster model of $\mathrm{Mp}$ infection ${ }^{14}$ and mouse models of $\mathrm{Mp}$ infection. ${ }^{15-17}$ A final score per mouse, both infected and uninfected, on a scale of 0 to 26 (least to most severe) was obtained based on assessment of the quantity and quality of peribronchiolar and peribronchial inflammatory infiltrates, luminal exudates, perivascular infiltrates, and parenchymal pneumonia.

\section{Neutrophil Elastase Activity Assay}

Neutrophil elastase activity in mouse BAL fluid samples and human neutrophil supernatants was measured using the substrate N-(methoxysuccinyl)-Ala-Ala-Pro-Val p-nitroanilide (Sigma-Aldrich) as previously described. ${ }^{18}$ In brief, the samples were mixed with an equal volume of $0.1 \mathrm{M}$ HEPES buffer containing $0.5 \mathrm{mmol} / \mathrm{L} \mathrm{NaCl}(\mathrm{pH} 7.5)$ and transferred to a 96-well plate. Saline solution mixed with buffer was used as a negative control. N-(methoxysuccinyl)-Ala-Ala-Pro-Val p-nitroanilide, $2 \mathrm{mmol} / \mathrm{L}$, was added to each well, incubated for 5 minutes, and measured for liberation of p-nitroaniline at $405 \mathrm{~nm}$. Neutrophil elastase activity was calculated using a human neutrophil elastase standard (Sigma-Aldrich) of known activity.

\section{Human Peripheral Blood Neutrophil Preparation and Stimulation}

Human neutrophils were obtained from three healthy donors in accordance with a protocol approved by the National Jewish Health Institutional Review Board. Human neutrophils were isolated using the plasma Percoll method as described previously. ${ }^{19}$ Neutrophils were suspended at $5 \times 10^{6} / \mathrm{mL}$ in Dulbecco's modified Eagle's medium supplemented with $10 \%$ fetal bovine serum and recombinant hSPLUNC1, $10 \mu \mathrm{g} / \mathrm{mL}$, or BSA, $10 \mu \mathrm{g} / \mathrm{mL}$. $\mathrm{Mp}, 10 \mathrm{CFU} / \mathrm{cell}$, was immediately added to neutrophils. Neutrophils were co-cultured with $\mathrm{Mp}$ for 2 hours at $37^{\circ} \mathrm{C}$, and supernatants were collected to quantify neutrophil elastase activity.

\section{Antibacterial Assay for Neutrophil Elastase}

The antibacterial effect of neutrophil elastase was tested by incubating $\mathrm{Mp}, 10^{5} \mathrm{CFU} / \mathrm{mL}$, with human sputum neutrophil elastase, $0,2,10$, or $50 \mu \mathrm{g} / \mathrm{mL}$ (Elastin Product Co., Inc, Owensville, MO), in a 96-well plate for 2 hours at $35^{\circ} \mathrm{C}$. The concentration of neutrophil elastase was within the range of sputum samples in patients with chronic lung disease. ${ }^{20-22} \mathrm{Mp}$ in the supernatants was plated on pleuropneumonia-like organism agar plates and incubated at $37^{\circ} \mathrm{C}, 5 \% \mathrm{CO}_{2}$, for 1 week to quantify Mp colonies.

\section{Generation of Recombinant hSPLUNC1 Protein}

Recombinant hSPLUNC1 protein was generated using a baculovirus protein expression system. The cDNA fragment encoding full-length human SPLUNC1 protein (256 amino acids) was amplified using PCR and ligated to pGEM-T Easy (Promega Corp., Madison, WI). The final clones were verified by sequencing, and subcloned into p479 insect cells expression vector. Sf9 cells were cotransfected with hSPLUNC1 cDNA and linearized baculovirus DNA. Cells were incubated at $27^{\circ} \mathrm{C}$ for 14 days, and supernatant containing SPLUNC1 carrying baculovirus was used to infect $\mathrm{Hi}-5$ cells. hSPLUNC1 protein from $\mathrm{Hi}-5$ cell culture was harvested and purified using a His-bind column (Novagen, Inc., Madison, WI). The purity of recombinant hSPLUNC1 protein was verified using SDS-polyacrylamide electrophoresis and Western blot analysis.

\section{Statistical Analysis}

Data were analyzed using commercially available software (PRISM; GraphPad Software, Inc., San Diego, CA). For normally distributed data, one-way analysis of variance was used for multiple comparisons, and Tukey's post hoc test was used where appropriate. The Student's $t$-test was used when only two groups were compared. Nonnormally distributed data were compared using the Wilcoxon rank sum test. $P<0.05$ was considered statistically significant.

\section{Results}

\section{Generation of C57BL/6 SPLUNC1 ${ }^{-1-}$ Mice}

Homologous recombination resulted in embryonic stem cell clones in which SPLUNC1 encoding exons 2 to 8 were replaced by the neomycin resistance gene (Figure 1, A and B). Loss of allele analysis confirmed that the targeting construct was inserted in the correct location on mouse chromosome 2 and in its entirety (Figure 1C), as previously described. ${ }^{11}$ Furthermore, unlike the SPLUNC $1^{+/+}$mice, SPLUNC1 $1^{-1-}$ mice did not express SPLUNC1 mRNA in the lung, including the trachea (Figure 1D). Likewise, SPLUNC1 $1^{-1-}$ mice did not secrete SPLUNC1 protein within BAL fluid (Figure 1E). SPLUNC1 ${ }^{-1-}$ mice were viable, fertile, and largely indistinguishable from SPLUNC1 ${ }^{+/+}$ littermates in general appearance, body weight, locomotion, and overt behavior.

\section{Characterization of FVB/n hSPLUNC1 Transgenic Mice}

Using quantitative real-time PCR, hSPLUNC1 mRNA was detected in transgene-positive mice but was undetectable in transgene-negative littermate control mice. Likewise, using a specific mouse anti-human 

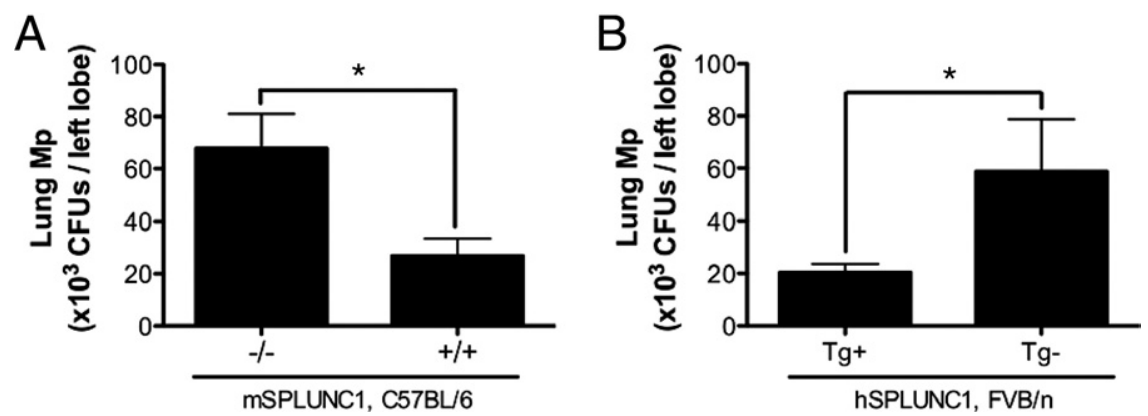

Figure 2. Lung Mp load in SPLUNC1 ${ }^{-/-}$and hSPLUNC1 transgenic mice. After 24 hours of infection, Mp load was quantified in lung tissue from $\mathrm{C} 57 \mathrm{BL} / 6 \mathrm{SPLUNC}^{-/-}$or SPLUNC1 $^{+/}$ mice ( $n=5$ mice per group) (A) or $\mathrm{FVB} / \mathrm{n}$ hSPLUNC1 transgene-positive $(\mathrm{Tg}+)$ or transgene-negative $(\mathrm{Tg}-)$ mice $(n=10$ mice per group) (B). A and $\mathbf{B}$, Data represent results obtained from three independent experiments. CFUs, colony-forming units. ${ }^{*} P<0.05$.
SPLUNC1 antibody, hSPLUNC1 was detectable only in transgene-positive mouse BAL fluid (Figure 1F). mSPLUNC1 mRNA levels were similar in both transgene-positive and transgene-negative mice.

\section{SPLUNC1 is Critical for in Vivo Lung Defense Against Mp Infection}

mSPLUNC1 knockout and hSPLUNC1 transgenic mouse models were used for dissection of the in vivo antimicrobial function of mSPLUNC1 and hSPLUNC1, respectively.

In the mSPLUNC1 knockout model (Figure 2A), Mp levels in SPLUNC1 ${ }^{-1-}$ C57BL/6 mouse lungs were threefold higher $(P<0.05)$ than those in the SPLUNC1 $1^{+/+}$ $\mathrm{C} 57 \mathrm{BL} / 6$ littermate control mice. Of note, in a separate experiment, $\mathrm{Mp}$ levels in lung homogenates were similar between SPLUNC1 ${ }^{-1-}$ and SPLUNC1 $1^{+/+}$mice at initiation of infection because entire lungs were obtained 10 minutes after bacterial inoculation.

In line with the knockout model, lung $\mathrm{Mp}$ load in FVB/n hSPLUNC1 transgenic mice was about threefold lower than that in the wild-type littermate control mice (Figure 2B). In vivo data explicitly demonstrated that SPLUNC1 is necessary and sufficient to confer host protection from $\mathrm{Mp}$ infection.

\section{Mechanisms Underlying SPLUNC1 Antimicrobial Functions}

To understand how SPLUNC1 may protect mice from Mp infection, the direct effects of hSPLUNC1 on Mp growth were tested. In a previous publication, the inhibitory effects of mSPLUNC1 on Mp growth were demonstrated. ${ }^{4}$ In the present study, previous findings were extended by performing an in vitro assay to directly assess the antimicrobial activity of hSPLUNC1 on Mp. Recombinant hSPLUNC1 protein also inhibited Mp growth compared with BSA (an irrelevant protein control) (Figure 3A).

Because $\mathrm{Mp}$ depends on adhesion to host epithelial cells for colonization and infection, ${ }^{23}$ it was hypothesized that SPLUNC1 may decrease in vivo Mp adherence to airway epithelium, thus promoting bacterial clearance from airways. Because P1 adhesin is one of the major Mp structural components involved in its adherence process, ${ }^{24,25}$ whether recombinant hSPLUNC1 protein inhibits $\mathrm{P} 1$ adhesin expression was tested. Co-incubation of $\mathrm{Mp}$ with recombinant hSPLUNC1 protein at a physiologic dose, $10 \mu \mathrm{g} / \mathrm{mL}$, for 24 hours (the same duration for mouse Mp infection) significantly decreased P1 adhesin mRNA levels compared with BSA (Figure 3B).

\section{Effect of SPLUNC1 on Lung Innate Inflammatory Responses to Mp Infection}

In the SPLUNC1 knockout mouse model, lung histopathologic features and the cellular profile in BAL fluid of saline solution-inoculated (control) SPLUNC1 ${ }^{-1-}$ and SPLUNC1 $1^{+/+}$mice were similar and mainly composed of macrophages. However, after Mp infection, Mpinfected SPLUNC1 ${ }^{-1-}$ mice demonstrated greater lung tissue inflammation as represented by a higher histopathology score than did the SPLUNC1 ${ }^{+/+}$mice (Figure 4A). SPLUNC1 ${ }^{-1-}$ mice exhibited enhanced peribronchial and perivascular leukocyte infiltration and pneumonia (Figure 4B). Similarly, BAL fluid from SPLUNC1 $1^{-1-}$ mice demonstrated a significant increase in neutrophils (Figure 4C) and total leukocytes. To examine neutrophil activation, neutrophil elastase activity in BAL fluid was measured.

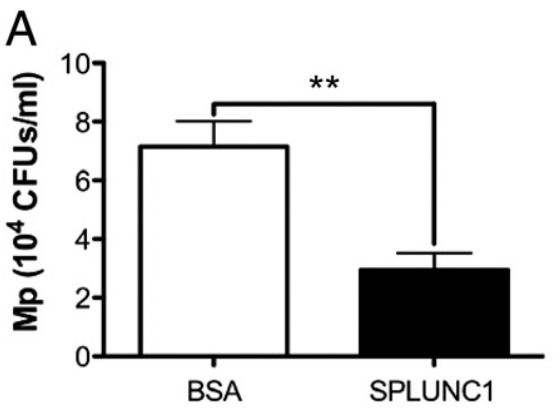

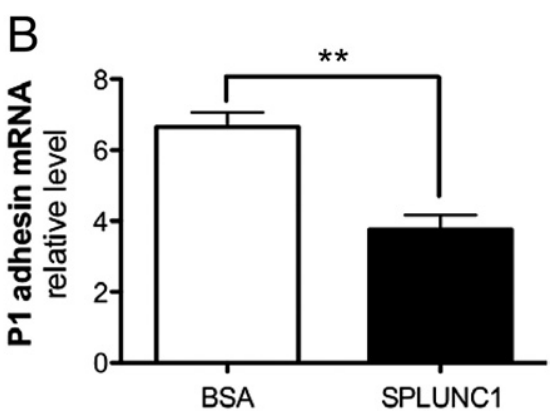

Figure 3. SPLUNC1 inhibits Mp growth and $\mathrm{P} 1$ adhesin mRNA. A: Recombinant hSPLUNC1 protein, $10 \mu \mathrm{g} / \mathrm{mL}$, significantly reduces $\mathrm{Mp}\left(10^{6}\right.$ CFUs/mL) growth when co-incubated in 96-well plates for 24 hours. B: Mp $\left(10^{6} \mathrm{CFUs} / \mathrm{mL}\right)$ was incubated with BSA, $10 \mu \mathrm{g} / \mathrm{mL}$, or recombinant hSPLUNC1, $10 \mu \mathrm{g} / \mathrm{mL}$, for 24 hours at $35^{\circ} \mathrm{C}$. Bacterial RNA was extracted, and quantitative realtime PCR was performed for P1 adhesin mRNA. $16 \mathrm{~S}$ was used as a housekeeping gene to normalize P1 adhesin expression. $\mathbf{A}$ and $\mathbf{B}$, Data represent results obtained from three independent experiments. CFUs, colony-forming units. ${ }^{\text {*** }} P<0.01$ 
A

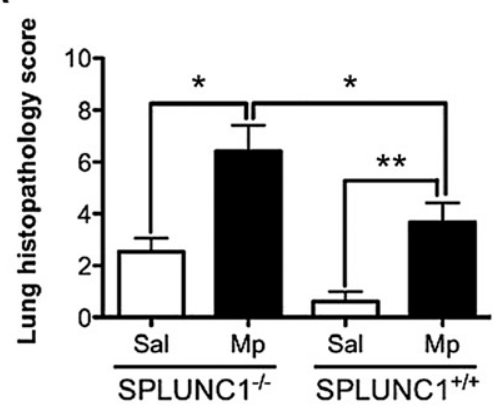

D

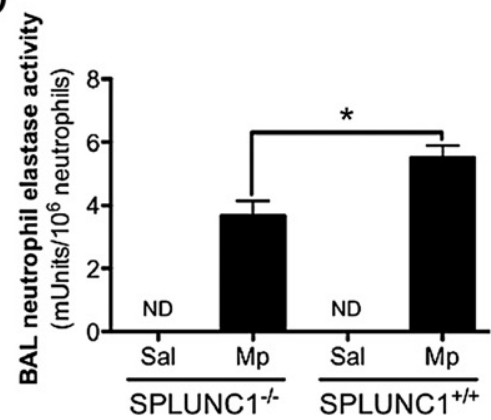

B

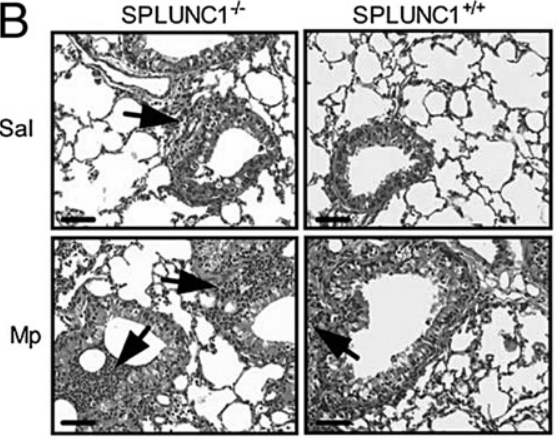

C

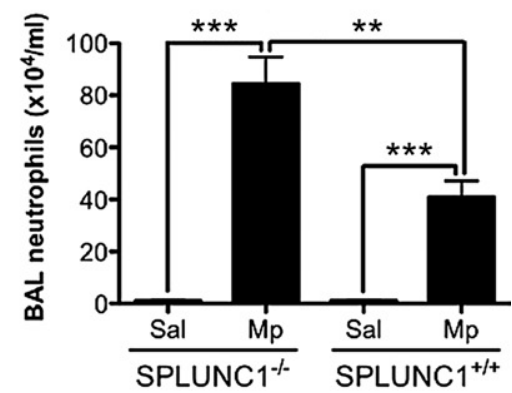

Figure 4. Effect of SPLUNC1 deficiency on lung inflammation after Mp infection. A: SPLUNC1 deficiency increases the severity of lung inflammation elicited by Mp. Paraffin sections $4-\mu \mathrm{m}$ thick were stained with H\&E, analyzed using light microscopy, and assigned a histopathology score in a blinded fashion. The groups consisted of saline solutiontreated (Sal) or Mp-infected (Mp) SPLUNC1 ${ }^{->-}$or SPLUNC1 ${ }^{+++}$mice $(n=5$ mice per group). B: Representative histopathologic microphotographs of lung tissues (original magnification, $\times 200$; scale bars $=100 \mu \mathrm{m}$ ). Upper left panel: Saline solution-treated SPLUNC1 ${ }^{-/-}$mice demonstrated minimal perivascular leukocyte infiltration (arrow). Bottom left panel: $\mathrm{Mp}$-infected SPLUNC1 ${ }^{-/-}$mice exhibited marked peribronchial and airway lumen leukocyte infiltration (arrows). Upper right panel: Saline solution-treated SPLUNC1 ${ }^{+/+}$mice exhibited no inflammation. Bottom right panel: Mp-infected SPLUNC1 ${ }^{+/+}$mice demonstrated moderate perivascular leukocyte infiltration (arrow). C: Neutrophil numbers in BAL fluid from saline solution-treated or Mp-infected SPLUNC1 $^{-/-}$or SPLUNC1 ${ }^{+/+}$mice $(n=5$ mice per group). D: Neutrophil elastase activity levels normalized to neutrophil numbers in BAL fluid from saline solution-treated or Mp-infected SPLUNC1 ${ }^{-/-}$or SPLUNC1 ${ }^{+/+}$mice ( $n=5$ mice per group). Data represent results obtained from three independent experiments. ${ }^{*} P<0.05,{ }^{* * *} P<$ $0.01,{ }^{* * * * *} P<0.001$.

Unlike the neutrophil number data, after $\mathrm{Mp}$ infection, neutrophil elastase activity normalized using neutrophil numbers was significantly lower in SPLUNC1 ${ }^{-1-}$ mice compared with littermate control mice (Figure 4D), which indicates that SPLUNC1 is important for stimulation of neutrophil elastase activity during the course of lung bacterial infection.

In the hSPLUNC1 transgenic model, in the absence of $\mathrm{Mp}$ infection, lung histopathologic features (Figures 5, A and $\mathrm{B}$ ) and neutrophil numbers (Figure 5C) were similar between transgene-positive and transgene-negative mice. In response to $\mathrm{Mp}$ infection, lung tissue inflammation was reduced in transgene-positive mice (Figure 5, A and $B$ ). In accordance with lung Mp levels (Figure 2), FVB/n wild-type mice demonstrated more BAL neutrophils and more severe pneumonia compared with C57BL/6 wild-type mice (Figure 4C and Figure 5C), indicating that the susceptibility to $\mathrm{Mp}$ infection varies in different strains of mice. Furthermore,

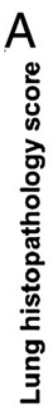
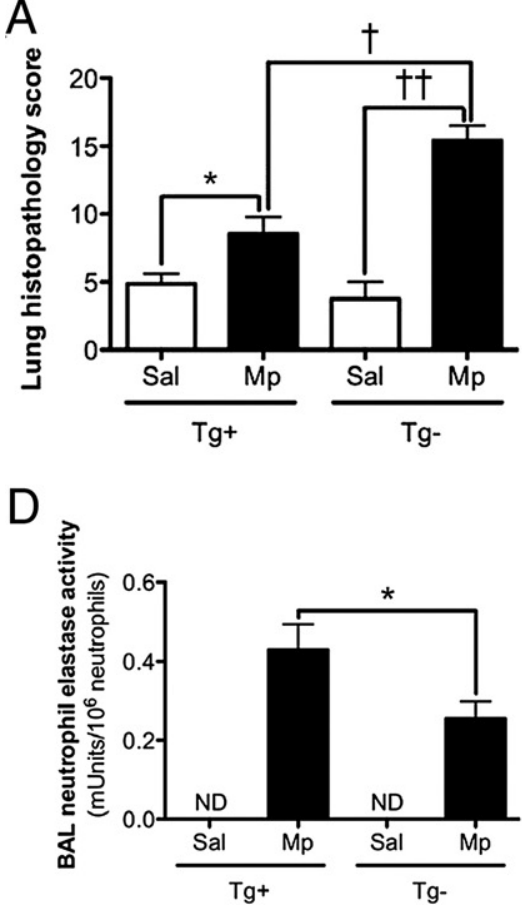

$\mathrm{B}$

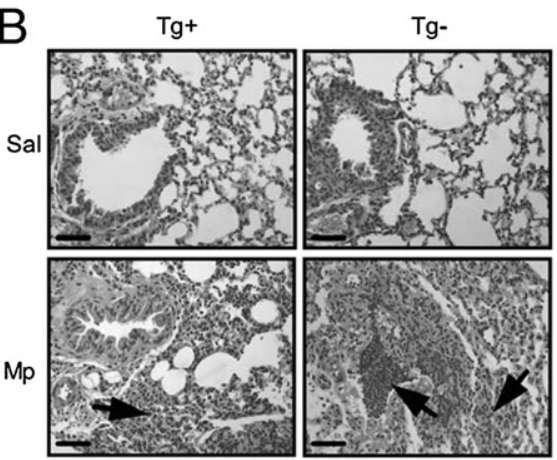

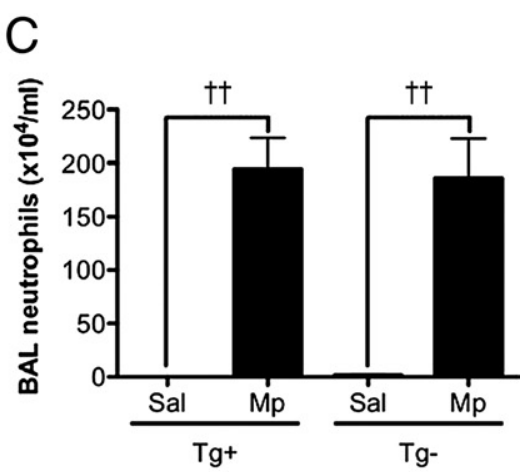

Figure 5. Effect of hSPLUNC1 overexpression on lung inflammation after $\mathrm{Mp}$ infection. $\mathbf{A}: \mathrm{hSPLUNC1}$ overexpression in mouse lungs decreases the severity of lung inflammation elicited by Mp. Paraffin sections $4-\mu \mathrm{m}$ thick were stained with H\&E, analyzed using light microscopy, and assigned a histopathology score in a blinded fashion. The groups consisted of saline solution-treated (Sal) or Mp-infected (Mp) hSPLUNC1 transgene-positive $(\mathrm{Tg}+)$ or transgene-negative $(\mathrm{Tg}-)$ mice $(n=10$ mice per group). B: Representative histopathologic microphotographs of lung tissues (original magnification $\times 200$; scale bars $=100 \mu \mathrm{m}$ ). Upper left panel: Saline solution-treated transgene-positive mice demonstrated no inflammation. Bottom left panel: Mp-infected transgene-positive mice exhibited moderate peribronchial leukocyte infiltration (arrow). Upper right panel: Saline solution-treated transgene-negative mice exhibited no inflammation. Bottom right panel: Mp-infected transgene-negative mice exhibited significant peribronchial and airway lumen leukocyte accumulation and severe pneumonia (arrows). C: Neutrophils in BAL fluid from saline solution-treated or Mp-infected transgene-positive or transgene-negative mice ( $n=10$ mice per group). D: Neutrophil elastase activity levels normalized to neutrophil numbers in BAL fluid from saline solution-treated or Mp-infected transgene-positive or transgenenegative mice ( $n=10$ mice per group). ND, not detected. ${ }^{*} P<0.05,{ }^{\dagger} P<0.005,{ }^{\dagger} P<0.0001$. 
A

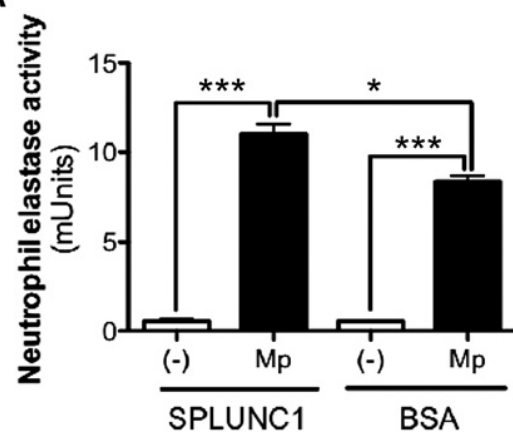

B

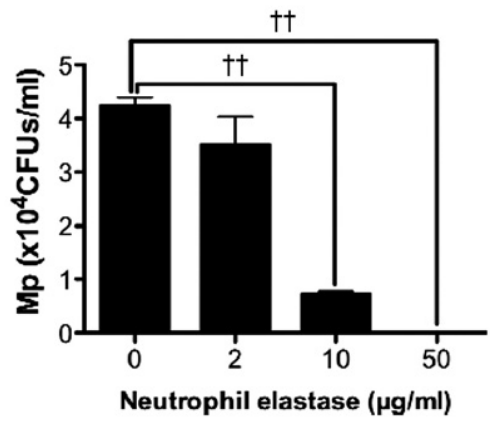

Figure 6. SPLUNC1 regulates human neutrophil activity. A: Recombinant hSPLUNC1 protein, 10 $\mu \mathrm{g} / \mathrm{mL}$, compared with BSA, $10 \mu \mathrm{g} / \mathrm{mL}$, enhanced elastase activity in peripheral human neutrophils after Mp infection for 2 hours. Data were obtained from three independent experiments. B: Human sputum neutrophil elastase markedly reduced Mp growth in a dose-dependent manner. Data were obtained from three independent experiments. CFUs, colony-forming units. ${ }^{*} P<0.05,{ }^{* * * * *} P<0.001,{ }^{\#} P<0.0001$ despite similar numbers of BAL neutrophils between transgene-positive and transgene-negative mice (Figure 5C), the transgene-positive mice demonstrated increased neutrophil elastase activity (Figure 5D).

\section{Mechanisms of Innate Inflammatory Response Regulation by SPLUNC1}

The data from mSPLUNC1 knockout and hSPLUNC1 transgenic models suggest that SPLUNC1 may affect lung leukocyte (eg, neutrophil) activation. In the context of in vivo bacterial infection, it is difficult to tease out the relative contribution of SPLUNC1 antimicrobial versus immunomodulatory effects to the final biological outcomes (eg, levels of leukocyte recruitment and functions). Therefore, to explicitly demonstrate the direct effect of SPLUNC1 on neutrophil activation, human peripheral blood neutrophils were treated with recombinant hSPLUNC1 protein or an irrelevant protein (ie, BSA) as a control, immediately followed by $\mathrm{Mp}$ infection for 2 hours. Mp infection stimulated neutrophil elastase activity, and the presence of SPLUNC1 further increased neutrophil elastase activity (Figure 6A). To test whether human neutrophil elastase is able to exert antimicrobial function against $\mathrm{Mp}, \mathrm{Mp}$ was incubated with human sputum neutrophil elastase. Neutrophil elastase markedly reduced $\mathrm{Mp}$ growth in a dose-dependent manner (Figure 6B).

\section{Discussion}

The present study revealed several novel aspects of SPLUNC1 functions. First, using SPLUNC1 knockout and transgenic mice, a critical in vivo role of SPLUNC1 was demonstrated in host defense against $\mathrm{Mp}$, a prominent respiratory pathogen. Second, it was observed that in the lung or primary cell cultures (eg, human peripheral blood neutrophils), SPLUNC1 moderately enhanced neutrophil elastase activity. These novel findings will provide invaluable insights in future studies with the objective of effectively eradicating bacterial (eg, $\mathrm{Mp}$ ) infections that persist in the airways of patients with chronic lung disease.

To our knowledge, this is the first study to report generation of SPLUNC1 ${ }^{-1-}$ mice and to test this model in bacterial infection. As previously reported, SPLUNC1 was highly expressed in the trachea and intrapulmonary airways in wild-type mice, and to a significantly lesser extent in other organs such as the thymus. ${ }^{26}$ SPLUNC1 was undetectable in any tissues or BAL fluid harvested from SPLUNC1 ${ }^{-1-}$ mice, which confirmed the gene knockout. Successful generation of SPLUNC1 ${ }^{-1-}$ mice provides a unique research approach to better define the in vivo SPLUNC1 function.

Use of SPLUNC1 ${ }^{-/-}$mice clearly demonstrated a significantly increased lung bacterial load in SPLUNC1 ${ }^{-1-}$ mice compared with wild-type mice. Although this finding is not surprising considering the previous in vitro SPLUNC1 functional study, one must be aware of the complexity and redundancy of innate immune defense mechanisms in vivo. For example, previous studies using surfactant proteins $A$ or $D$ or $\beta$-defensin knockout mice have demonstrated impaired bacterial clearance from the lung. ${ }^{27-29}$ In addition, airway epithelial cells produce several other major host defense molecules such as lactotransferrin. ${ }^{30}$ The present results of impaired Mp clearance from SPLUNC1 ${ }^{-1-}$ mouse lungs advance the lung innate immunity field by demonstrating the in vivo function of SPLUNC1. Furthermore, it was demonstrated that hSPLUNC1 inhibited Mp growth, thus extending previous results using mouse SPLUNC1 protein. The present study also suggests that SPLUNC1, by down-regulating the expression of Mp P1 adhesin, may prevent Mp adhesion to lung epithelial cells. SPLUNC1 ${ }^{-1-}$ primary airway ep-

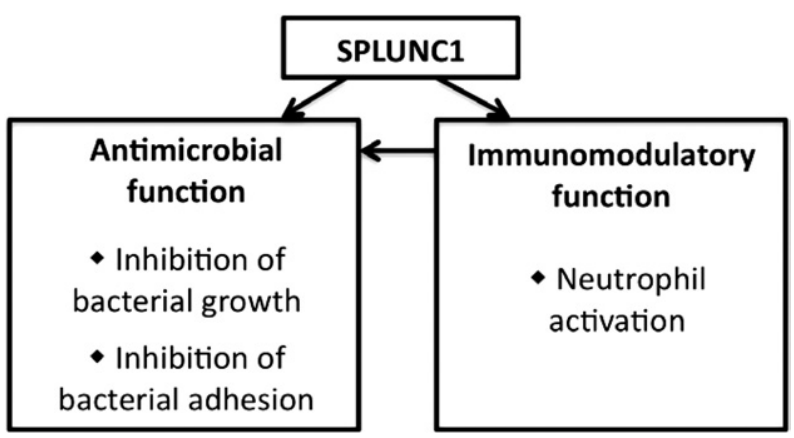

Figure 7. Antimicrobial and immunomodulatory functions of SPLUNC1. SPLUNC1 exerts antibacterial effects via inhibition of bacterial growth or inhibition of bacterial adhesion. Furthermore, SPLUNC1 enhances host defense against bacterial infection indirectly through increasing neutrophil activation. In turn, activated neutrophils participate in elimination of the microorganisms via release of neutrophil elastase. 
ithelial cells exhibited higher (about threefold increase) levels of P1 adhesin mRNA compared with SPLUNC1 ${ }^{+/+}$ cells when cultured at the air-liquid interface for 10 days followed by Mp infection for 24 hours. Nevertheless, the antimicrobial mechanisms of SPLUNC1 protein remain unclear. Although findings of the present study suggest $\mathrm{P} 1$ adhesin inhibition as one of the mechanisms, it is conceivable that SPLUNC1 may affect other molecules such as other Mp adhesins (eg, P30). Future experiments will be required to dissect the broader effects of SPLUNC1 on Mp biology.

How SPLUNC1 exerts its host defense function in vivo remains largely unclear. SPLUNC1 $1^{-1-}$ mice were viable and visually indistinguishable from wild-type littermate control mice if left untreated. However, when SPLUNC1 $1^{-1-}$ mice were infected with $\mathrm{Mp}$, they not only demonstrated impaired lung bacterial clearance but also reduced neutrophil activation. Although neutrophils are clearly implicated in killing gram-negative or gram-positive bacteria $^{31,32}$ the role of neutrophils or neutrophil elastase in $\mathrm{Mp}$ infection is not clear. To our knowledge, the present study for the first time demonstrated enhancement of human neutrophil elastase activity by SPLUNC1. Moreover, neutrophil elastase directly inhibited $\mathrm{Mp}$ growth. However, neutrophil elastase also causes lung tissue damage including emphysema. ${ }^{33}$ The present data demonstrated higher neutrophil elastase activity but less histologic alterations in the wild-type mice. This could be explained by the shorter duration (24 hours) of the mouse model in which neutrophil elastase antimicrobial function may predominate or overpower the tissue damaging effects of neutrophil elastase. Long-term mouse models will be required to further address the balance of beneficial versus detrimental functions of neutrophil elastase in SPLUNC1 knockout mice. Together, the data suggest that in addition to its direct antibacterial effect, SPLUNC1 modulates the host innate immune response (eg, enhancing neutrophil activation), thereby explaining its in vivo host defense functions. In addition to enhancing neutrophil elastase activity, as demonstrated in preliminary studies, SPLUNC1 may also increase neutrophil chemotaxis and IL-8 secretion after TLR2 agonist stimulation. Whether SPLUNC1 exerts similar function in other cells such as macrophages awaits future study. Similarly, the molecular mechanisms of SPLUNC1 immunomodulatory function on neutrophils remain to be investigated.

One unique aspect of the present study is that not only was the in vivo function of MSPLUNC1 revealed in the knockout model, but the transgenic mouse model was used to exclusively overexpress hSPLUNC1 in airway epithelium. hSPLUNC1 overexpression in mouse airways resulted in doubling of SPLUNC1 protein in BAL fluid and significantly prevented $\mathrm{Mp}$ infection and associated lung inflammation. These findings suggest that hSPLUNC1 and mSPLUNC1 share similar antibacterial and immunomodulatory functions. Therefore, SPLUNC1-deficient and hSPLUNC1-transgenic mouse models can be used to further explore the function of SPLUNC1 in infection of other species of bacteria under human disease conditions such as asthma, chronic obstructive pulmonary disease, and cystic fibrosis. These chronic lung diseases are characterized by persistent bacterial infection in the airways and reduced SPLUNC1 levels (eg, chronic obstructive pulmonary disease and rhinitis). ${ }^{34,35}$ In addition to $\mathrm{Mp}$, several other species of bacteria including nontypeable Haemophilus influenzae, Streptococcus pneumoniae, and Moraxella catarrhalis have been detected in the airways in chronic lung diseases. ${ }^{36-38}$ Whether SPLUNC1 also exerts antibacterial effects on these additional strains of bacteria is in question and is currently being investigated. Furthermore, revealing how SPLUNC1 modulates the function of innate immune cells such as neutrophils and macrophages may be important. Understanding various aspects of SPLUNC1 functions will help in designing novel therapies to enhance host endogenous antimicrobial responses that typically are not associated with drug resistance.

In summary, SPLUNC1 has antibacterial effects by inhibiting Mp growth and inhibiting bacterial adherence (Figure 7). In addition, SPLUNC1 indirectly enhances host defenses against bacterial infection through modulation of neutrophil activation, which in turn may kill the bacteria.

\section{Acknowledgments}

We thank Dr. Magnus Nord, M.D., Ph.D. (Karolinska Institute, Stockholm, Sweden), for supplying the genomic fragment, and Dr. John Kappler, Ph.D. (National Jewish Health), for donating the p479 insect cells expression vector; Fran Crawford and Fangkun Ning for technical assistance in generating recombinant hSPLUNC1 protein; Dr. Gongyi Zhang, Ph.D., for thoughtful discussions; and Dr. Jennifer Matsuda, Ph.D., and James T. Gross (Mouse Genetics Core facility, National Jewish Health) for expert technical assistance.

\section{References}

1. Akira S, Uematsu S, Takeuchi O: Pathogen recognition and innate immunity. Cell 2006, 124:783-801

2. Bals R, Hiemstra PS: Innate immunity in the lung: how epithelial cells fight against respiratory pathogens. Eur Respir J 2004, 23:327-333

3. Bingle CD, Bingle L: Characterisation of the human PLUNC gene, a gene product with an upper airways and nasopharyngeal restricted expression pattern. Biochim Biophys Acta 2000, 1493:363-367

4. Chu HW, Thaikoottathil J, Rino JG, Zhang G, Wu Q, Moss T, Refaeli Y, Bowler R, Wenzel SE, Chen Z, Zdunek J, Breed R, Young R, Allaire E, Martin RJ: Function and regulation of SPLUNC1 protein in Mycoplasma infection and allergic inflammation. J Immunol 2007, 179: 3995-4002

5. Bingle CD, Gorr SU: Host defense in oral and airway epithelia: chromosome 20 contributes a new protein family. Int J Biochem Cell Biol 2004, 36:2144-2152

6. Zhou HD, Li XL, Li GY, Zhou M, Liu HY, Yang YX, Deng T, Ma J, Sheng SR: Effect of SPLUNC1 protein on the Pseudomonas aeruginosa and Epstein-Barr virus. Mol Cell Biochem 2008, 309:191-197

7. Garcia-Caballero A, Rasmussen JE, Gaillard E, Watson MJ, Olsen JC Donaldson SH, Stutts MJ, Tarran R: SPLUNC1 regulates airway surface liquid volume by protecting ENaC from proteolytic cleavage. Proc Natl Acad Sci USA 2009, 106:11412-11417

8. Talkington DF, Waites KB, Schwartz SB, Besser RE: Emerging from obscurity: understanding pulmonary and extrapulmonary syndromes, pathogenesis, and epidemiology of human Mycoplasma pneumoniae infections. Emerging Infections, ed 5. Edited by WM Scheld, WA Craig, JM Hughes. Washington, DC: ASM Press, 2001, pp 57-84 
9. Kraft M, Cassell GH, Henson JE, Watson H, Williamson J, Marmion BP, Gaydos CA, Martin RJ: Detection of Mycoplasma pneumoniae in the airways of adults with chronic asthma. Am J Respir Crit Care Med 1998, 158:998-1001

10. Martin RJ, Kraft M, Chu HW, Berns EA, Cassell GH: A link between chronic asthma and chronic infection. J Allergy Clin Immunol 2001, 107:595-601

11. Valenzuela DM, Murphy AJ, Frendewey D, Gale NW, Economides AN, Auerbach W, Poueymirou WT, Adams NC, Rojas J, Yasenchak J, Chernomorsky R, Boucher M, Elsasser AL, Esau L, Zheng J, Griffiths JA, Wang X, Su H, Xue Y, Dominguez MG, Noguera I, Torres R, Macdonald LE, Stewart AF, DeChiara TM, Yancopoulos GD: Highthroughput engineering of the mouse genome coupled with highresolution expression analysis. Nature Biotechnol 2003, 21:652-659

12. Wu Q, Martin RJ, Rino JG, Jeyaseelan S, Breed R, Chu HW: A deficient TLR2 signaling promotes airway mucin production in Mycoplasma pneumoniae-infected allergic mice. Am J Physiol Lung Cell Mol Physiol 2007, 292:L1064-L1072

13. Numata M, Chu HW, Dakhama A, Voelker DR: Pulmonary surfactant phosphatidylglycerol inhibits respiratory syncytial virus-induced inflammation and infection. Proc Natl Acad Sci USA 2010, 107:320-325

14. Cimolai N, Taylor GP, Mah D, Morrison BJ: Definition and application of a histopathological scoring scheme for an animal model of acute Mycoplasma pneumoniae pulmonary infection. Microbiol Immunol 1992, 36:465-478

15. Hardy RD, Jafri HS, Olsen K, Hatfield J, Iglehart J, Rogers BB, Pate P, Cassell G, McCracken GH, Ramilo O: Mycoplasma pneumoniae induces chronic respiratory infection, airway hyperreactivity, and pulmonary inflammation: a murine model of infection-associated chronic reactive airway disease. Infect Immun 2002, 70:649-654

16. Martin RJ, Chu HW, Honour JM, Harbeck RJ: Airway inflammation and bronchial hyperresponsiveness after Mycoplasma pneumoniae infection in a murine model. Am J Respir Cell Mol Bio 2001, 24:577-582

17. Chu HW, Honour JM, Rawlinson CA, Harbeck RJ, Martin RJ: Effects of respiratory Mycoplasma pneumoniae infection on allergen-induced bronchial hyperresponsiveness and lung inflammation in mice. Infect Immun 2003, 71:1520-1526

18. Bergsson G, Reeves EP, McNally P, Chotirmall SH, Greene CM, Greally P, Murphy P, O'Neill SJ, McElvaney NG: LL-37 complexation with glycosaminoglycans in cystic fibrosis lungs inhibits antimicrobial activity, which can be restored by hypertonic saline. J Immunol 2009 , 183:543-551

19. Haslett C, Guthrie LA, Kopaniak MM, Johnston RB Jr, Henson PM: Modulation of multiple neutrophil functions by preparative methods or trace concentrations of bacterial lipopolysaccharide. Am J Pathol 1985, 119:101-110

20. Poncz L, Jentoft N, Ho MC, Dearborn DG: Kinetics of proteolysis of hog gastric mucin by human neutrophil elastase and by Pseudomonas aeruginosa elastase. Infect Immun 1988, 56:703-704

21. Grootendorst DC, Gauw SA, Verhoosel RM, Sterk PJ, Hospers JJ, Bredenbroker D, Bethke TD, Hiemstra PS, Rabe KF: Reduction in sputum neutrophil and eosinophil numbers by the PDE4 inhibitor roflumilast in patients with COPD. Thorax 2007, 62:1081-1087

22. Kelly MG, Brown V, Martin SL, Ennis M, Elborn JS: Comparison of sputum induction using high-output and low-output ultrasonic nebulizers in normal subjects and patients with COPD. Chest 2002, 122 : 955-959

23. Rottem S: Interaction of mycoplasmas with host cells. Physiol Rev 2003, 83:417-432

24. Inamine JM, Denny TP, Loechel S, Schaper U, Huang CH, Bott KF, Hu PC: Nucleotide sequence of the P1 attachment-protein gene of Mycoplasma pneumoniae. Gene 1988, 64:217-229

25. Razin S, Jacobs E: Mycoplasma adhesion. J Gen Microbiol 1992 138:407-422

26. LeClair EE, Nguyen L, Bingle L, MacGowan A, Singleton V, Ward SJ, Bingle CD: Genomic organization of the mouse PLUNC gene and expression in the developing airways and thymus. Biochem Biophys Res Commun 2001, 284:792-797

27. LeVine AM, Kurak KE, Bruno MD, Stark JM, Whitsett JA, Korfhagen TR: Surfactant protein-A-deficient mice are susceptible to Pseudomonas aeruginosa infection. Am J Respir Cell Mol Biol 1998, 19:700-708

28. LeVine AM, Whitsett JA, Gwozdz JA, Richardson TR, Fisher JH, Burhans MS, Korfhagen TR: Distinct effects of surfactant protein A or D deficiency during bacterial infection on the lung. J Immunol 2000, 165:3934-3940

29. Moser C, Weiner DJ, Lysenko E, Bals R, Weiser JN, Wilson JM: Beta-defensin 1 contributes to pulmonary innate immunity in mice. Infect Immun 2002, 70:3068-3072

30. Sagel SD, Sontag MK, Accurso FJ: Relationship between antimicrobial proteins and airway inflammation and infection in cystic fibrosis. Pediatr Pulmonol 2009, 44:402-409

31. Belaaouaj A: Neutrophil elastase-mediated killing of bacteria: lessons from targeted mutagenesis. Microbes Infect 2002, 4:1259-1264

32. Brinkmann V, Reichard U, Goosmann C, Fauler B, Uhlemann Y, Weiss DS, Weinrauch $Y$, Zychlinsky A: Neutrophil extracellular traps kill bacteria. Science 2004, 303:1532-1535

33. Lungarella G, Cavarra E, Lucattelli M, Martorana PA: The dual role of neutrophil elastase in lung destruction and repair. Int J Biochem Cell Biol 2008, 40:1287-1296

34. Ghafouri B, Irander K, Lindbom J, Tagesson C, Lindahl M: Comparative proteomics of nasal fluid in seasonal allergic rhinitis. J Proteome Res 2006, 5:330-338

35. Steiling K, Lenburg ME, Spira A: Airway gene expression in chronic obstructive pulmonary disease. Proc Am Thorac Soc 2009, 6:697-700

36. Murphy TF, Brauer AL, Schiffmacher AT, Sethi S: Persistent colonization by Haemophilus influenzae in chronic obstructive pulmonary disease. Am J Respir Crit Care Med 2004, 170:266-272

37. Wilson R: Evidence of bacterial infection in acute exacerbations of chronic bronchitis. Semin Respir Infect 2000, 15:208-215

38. Kraft M: The role of bacterial infections in asthma. Clin Chest Med 2000, 21:301-313 